\title{
EXPLORING THE POTENTIAL OF FULL WAVEFORM AIRBORNE LIDAR FEATURES AND ITS FUSION WITH RGB IMAGE IN CLASSIFICATION OF A SPARSELY FORESTED AREA
}

\author{
Masoud Babadi ${ }^{1 *}$, Mehran Sattari $^{1}$, Siavash Iran Pour ${ }^{1,2}$ \\ ${ }^{1}$ Department of Geomatics Engineering, Faculty of Civil Engineering and Transportation, University of Isfahan, Isfahan, Iran - \\ (masoud.babadi92@trn, sattari@eng)ui.ac.ir, iranpour.siavash@gmail.com \\ ${ }^{2}$ Institute of Geodesy, University of Stuttgart, Stuttgart, Germany \\ siavash.iran-pour@gis.uni-stuttgart.de
}

KEY WORDS: Forest Management, Full Waveform LiDAR, Aerial Image, Classification, Tree species, Data Fusion

\begin{abstract}
:
Precise measurements of forest trees is very important in environmental protection. Measuring trees parameters by use of groundbased inventories is time and cost consuming. Employing advanced remote sensing techniques to obtain forest parameters has recently made a great progress step in this research area. Among the information resources of the study field, full waveform LiDAR data have attracted the attention of researchers in the recent years. However, decomposing LiDAR waveforms is one of the challenges in the data processing. In fact, the procedure of waveform decomposition causes some of the useful information in waveforms to be lost. In this study, we aim to investigate the potential use of non-decomposed full waveform LiDAR features and its fusion with optical images in classification of a sparsely forested area. We consider three classes including i) ground, ii) Quercus wislizeni and iii) Quercus douglusii for the classification procedure. In order to compare the results, five different strategies, namely i) RGB image, ii) common LiDAR features, iii) fusion of RGB image and common LiDAR features, iv) LiDAR waveform structural features and v) fusion of RGB image and LiDAR waveform structural features have been utilized for classifying the study area. The results of our study show that classification via using fusion of LiDAR waveform features and RGB image leads to the highest classification accuracy.
\end{abstract}

\section{INTRODUCTION}

Recently, the importance of preserving the environment is universally acknowledged (Foody, 2003; Giuliani et al., 2017). Precise measurements of the environment and especially obtaining parameters of forest trees by use of ground-based inventories is an exhausting job which is both time and cost consuming. During the last decades, developing automatic method became an important concern for many researchers in this field of study (Katila and Tomppo, 2001; Suárez et al., 2005). The advent of remote sensing techniques and data availability in the recent years have significantly revolutionised many study fields in environment monitoring, among them, tree species classification. Nowadays, aerial or satellite imagery is one of the main remote sensing information sources for this purpose (Brandtberg, 2002; Fassnacht et al., 2016; Immitzer et al., 2012). Another remote sensing information resources which has attracted the attention of many researchers in the last two decades is LiDAR data. The high performance of LiDAR in monitoring the vertical structure of trees provides reliable information for obtaining the forests parameters. That is especially the case for full waveform LiDAR data which can record returned energy of the objects (Mallet and Bretar, 2009; Reitberger et al., 2006). However, decomposing LiDAR waveforms is one of the biggest challenges in processing the data (Chauve et al., 2008; Mallet and Bretar, 2009). In fact, in many applications such as single tree segmentation, LiDAR waveform needs to be decomposed for further processing, but the procedure of waveform decomposition itself causes some of the waveform information to be lost (Vaughn et al., 2011, 2012).

In most of classification tasks, LiDAR waveforms are decomposed to extract point cloud and some conventional features such as amplitude and intensity where these data are used for classification in the next steps. In (Reitberger et al., 2008), authors have extracted three-dimensional (3D) point clouds and LiDAR features like the intensity and the pulse width from laser beam reflections by the use of fitting a series of Gaussian models to the waveform pulses. Afterwards, tree crowns were delineated using the canopy height model (CHM) and were classified into coniferous and deciduous classes with overall accuracy of $85 \%$ in a leaf-on and $96 \%$ in a leaf-off situation. Heinzel and Koch (2011) have identified three most important variables in their work based on the intensity, the width and the total number of targets and classified six, four and two tree species with an overall accuracy of 57\%,78\% and 91\%, respectively. Yao et al. (2012) detected single trees automatically by a 3D segmentation technique. In order to classify trees, they used salient features that are defined on single 3D tree segments and the additional information extracted from the reflected laser signal by the waveform decomposition. Finally, deciduous and coniferous trees were classified with $93 \%$ and $95 \%$ accuracy with an unsupervised and supervised classifier, respectively. Cao et al. (2016) have extracted full-waveform metrics by a voxel-based composite waveform approach and examined Random-Forests classifier to discriminate six subtropical tree species. One of the main problems of these methods is smoothing and decomposing waveforms to extract point cloud and features for classification tasks which cause losing important information of waveforms and their potential use for classification (Vaughn et al., 2011).

To the best of our knowledge, a few experiments have been done for forested area classification using integration of nondecomposed full waveform LiDAR data and aerial imagery. In this study, we intend to explore the potential employment of such data and in particular structural features of full waveform LiDAR data in classification of a sparsely forested area. To this end, some structural features have been extracted from full waveform

\footnotetext{
* Corresponding author
} 
airborne LiDAR data and have been integrated with an aerial RGB image to classify the study area. In order to compare the potential of using this data, we apply and assess five different strategies including i) using RGB image, ii) common LiDAR features, iii) fusion of RGB image and common LiDAR features, iv) structural features of full waveform LiDAR and v) fusion of RGB image and structural features of full waveform LiDAR for classifying the study area. For studying the effect of normalization on classification using each data source, classification with and without normalizing the features has been also performed.

The rest of the paper is organized as follows. In section 2, the study area and the data sources are introduced. In the third section, the methodology for classifying study area using each dataset and extracting structural features of full waveform LiDAR is elaborated. Section 4 involves with describing the experimental results. Finally, our conclusion is given in section 5.

\section{DATASET}

\subsection{Study Area}

The proposed method in this study was evaluated on airborne full waveform LiDAR data and RGB aerial imagery of Madera experimental range in California which has been collected through the National Ecological Observation Network (NEON) project. This study area extents from $119.735 \mathrm{~W}$ to $119.738 \mathrm{~W}$ and from $37.111 \mathrm{~N}$ to $37.113 \mathrm{~N}$, covering an area of about 6.25 ha and is an open woodland dominated by Quercus wislizeni and Quercus douglasii with scattered shrubs and a nearly continuous cover of herbaceous plants.

\subsection{LiDAR Data}

The LiDAR data used in this study was collected by an Optech Gemini LiDAR system at a nominal range of $1000 \mathrm{~m}$ (which means the aircraft flew at $1000 \mathrm{~m} \mathrm{AGL})$, wide divergence $(0.8$ $\mathrm{mrad}), 100 \mathrm{kHz}$ PRF, and a mirror scan frequency of $50 \mathrm{~Hz}$ and full scan angle of 37 degrees. This achieves a nominal 3.82 points (first return) per meter squared at Nadir with a $0.8 \mathrm{~m}$ diameter spot and a spot spacing of about $0.524 \mathrm{~m}$ in the across-track direction and $0.5 \mathrm{~m}$ in the along-track direction. The outgoing pulse width is about 15 nano-second and the waveform is digitized with 1 nano-second bins and a 12 bit analog to digital converter (ADC). In order to collect this data, the aircraft was flying either North-to-South or South-to-North where two flight lines of LiDAR data were provided in an area of $250 \mathrm{~m} \times 250 \mathrm{~m}$. Finally, 258667 waveforms have been recorded by this system. The first return point cloud of this data is illustrated in Figure 1(a).

\subsection{Image Data}

The aerial image exploited in this study has been captured in three bands (red, green and blue) simultaneously with LiDAR data collection. The image pixel size is $50 \mathrm{~cm}$ and its dimension is $1000 \times 1000$ pixels. The areal image of the study area in shown in Figure 1(b).

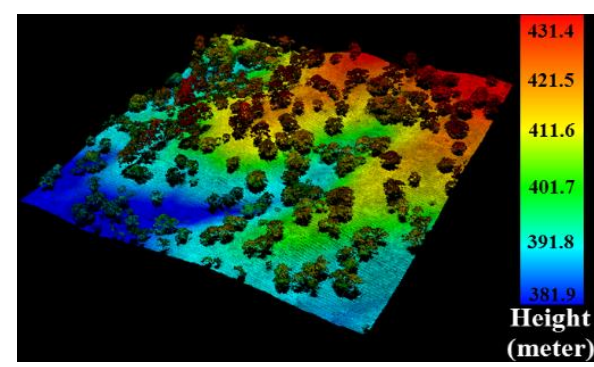

(a)

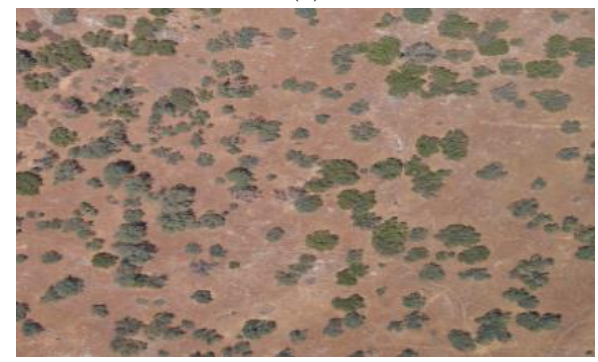

(b)

Figure 1. (a) First return point cloud (the legend shows points altitude from the sea level) and (b) aerial image of the study area.

\section{METHODOLOGY}

The focus of this study is on investigating the potential use of non-decomposed LiDAR waveform features and its fusion with RGB image in classifying a forested area. Thereafter, we aim to compare the results of our strategy with the results of the other methods. For this reason, minimum distance (MD) as a simple, fast and widely-used classifier is being employed for the classification. That is because MD has lower computational burden due to its simplicity and is free parameter when it is compared with many other cutting-edge classifiers such as SVM (Boiman et al., 2008; Lim et al., 2000; Zhang et al., 2006). Most of trees in the study area (90\%) are oak trees (Quercus wislizeni and Quercus douglasii) and as a results, three classes considered in this study including ground, Quercus wislizeni and Quercus douglusii classes. The ground truth (GT) used in this study was produced using the reported position of a limited number of trees (16 trees) and visual interpretation of the RGB image and multiple high-resolution satellite images of the study area. In this study, $60 \%$ of the GT was considered as training data where the rest of data was used as test data. The GT used in this study is illustrated in Figure 2.

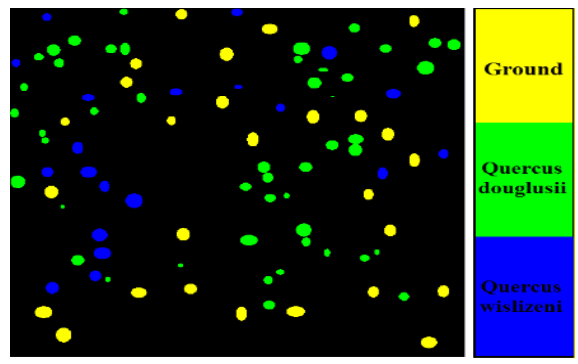

Figure 2. The GT used in the study for classification.

As mentioned, we use different strategies in this study for the classification procedure. The methods are briefly presented in the following. 


\subsection{Classification using aerial image (RGB)}

In this case, the study area was classified just using three bands (red, green and blue) of the aerial image.

\subsection{Classification using common LiDAR features related to the first return LiDAR pulse (first return)}

Here, the corresponding LiDAR points to each pixel are identified and the amplitude, width and intensity of the first peak (first return) along with the total number of peaks in the waveform corresponding to each point are extracted and used as common features for classification. The reason for using the amplitude, width and intensity of the only first return for classification is that the first peak in each waveform is the most trustable among the waveform peaks, where in the others, parts of laser energy are being reduced and affected by the previous objects in the laser path (Abed, 2012; Hyyppä et al., 2009).

\subsection{Classification using fusion of common LiDAR features and aerial image (RGB and first return)}

In this case, common LiDAR features along with the three bands of the aerial image are employed for classification.

\subsection{Classification using structural features of full waveform LiDAR (waveform)}

Here, the corresponding LiDAR points to each pixel are identified and LiDAR waveforms related to each point are extracted. Afterwards, without decomposing waveforms, eight structural features are extracted from raw LiDAR waveforms and used for classification. These features are introduced in the following:

\subsubsection{Integration of waveform}

Integration of waveform can be physically defined as a summation of all intensities in waveform which is affected by parameters such as specification of existed objects in laser path and their distances to each other. This feature, therefore, in the case of trees is different and depends on the reflectivity, density and spatial arrangement of the leaves and branches. The feature is defined as a summation of values of waveform amplitude which can be expressed as equation (1):

$$
P I=\sum_{i=1}^{n} x_{i}
$$

where $x_{i}$ is the energy of waveform in ith nano-second and $n$ is wavelength. A sample of this feature is shown in Figure 3(a).

\subsubsection{Amplitude of highest peak}

This feature is defined as the amplitude of highest detected peak in each waveform and is the return energy estimate of the corresponding object in laser path which is affected by type shape and specification of the object. A sample of this feature is illustrated in Figure 3(b). The feature has been used in many studied (Hollaus et al., 2009; Lindberg et al., 2014)

\subsubsection{Location of highest peak}

This feature is defined as the time that the highest potential peak is recorded which corresponds to the distance between the position of the object (identified by the highest detected peak) and the beginning of the wave recording. The feature is affected by parameters such as the object specification and distances between the detected objects in the laser path. Figure 3(b) illustrates this feature for a sample waveform.

\subsubsection{Distance between first and last detected peak}

In this method, the feature is defined as interval time distance between first and last detected peaks on horizontal axis of waveform diagram which is corresponded to the distance between the first and last object in the laser path (Cao et al., 2016). For some of waveforms related to trees, this feature can be considered as tree height if the laser is shot vertically and reaches the ground. This feature can play an important role in waveform classification. A sample of this feature is shown in Figure 3(b).

\subsubsection{Width to height ratio of first peak}

Width to height ratio of first peak is defined as width of estimated Gaussian model of first potential detected peak at $\% 50$ of its amplitude to its amplitude ratio. This feature is affected by some parameters such as type and shape of first object in laser path and for example, for the sample waveform illustrated in Figure 3(c) is $\frac{W 1}{A 1}$ which $W 1$ and $A 1$ are the width and amplitude of first detected peak, respectively. Amplitude and width of detected peaks in waveforms have been used in many classification tasks like (Reitberger et al., 2008) (Hollaus et al., 2009) .

\subsubsection{Sum of width to height ratios}

This feature is defined as sum of estimated width (using Gaussian function) to height ratios of all detected potential peaks in a waveform and physically relates to both fundamental parameters (amplitude and width) of all peaks. For the sample waveform shown in Figure 3(c) the value of this feature is $\frac{W 1}{A 1}+\frac{W 2}{A 2}+$ $\frac{W 3}{A 3}+\frac{W 4}{A 4}$ which $W i$ and $A i$ are width and amplitude of ith detected peak. This feature is affected by location, shape and type of objects existing in laser path.

\subsubsection{Intensity of first detected peak}

The intensity of first detected peak is used in many segmentation and classification tasks (Reitberger et al., 2009), (Hovi et al., 2016) and is defined as the intensity of first detected peak in a waveform. A sample of this feature is illustrated in Figure 3(d). The intensity of first detected peak is equal to the area under the waveform curve of first peak which is estimated by (Reitberger et al., 2008):

$$
I_{i}=\sqrt{2 * \pi} * A_{1} * \sigma_{1}
$$

where $A_{1}$ is the amplitude and $\sigma_{1}$ is standard deviation of the estimated Gaussian function of the first peak.

\subsubsection{First energy drop}

First energy drop is defined as the location that a temporary energy drop occurs in the waveform before hitting to the last object in the laser path where its value in the case of waveforms which do not have this energy drop is considered as zero. This feature is affected by characteristics and distances between objects. A sample of this feature is illustrated in Figure 3(e).

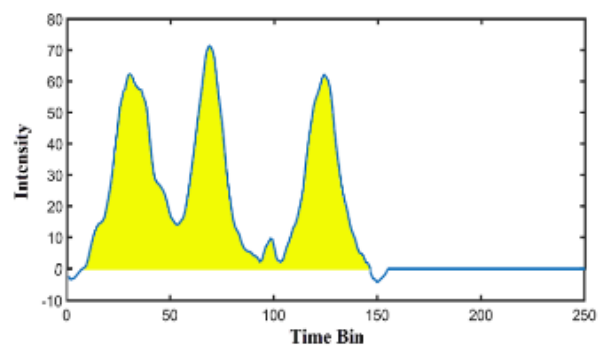


(a)

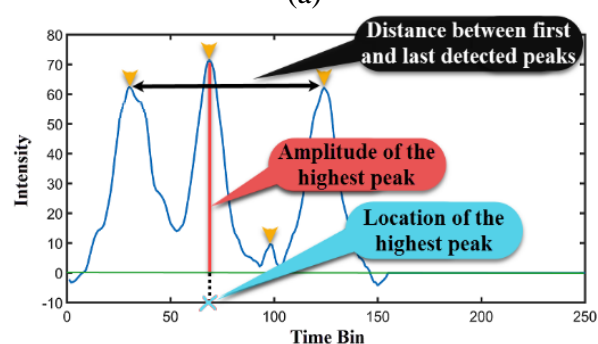

(b)

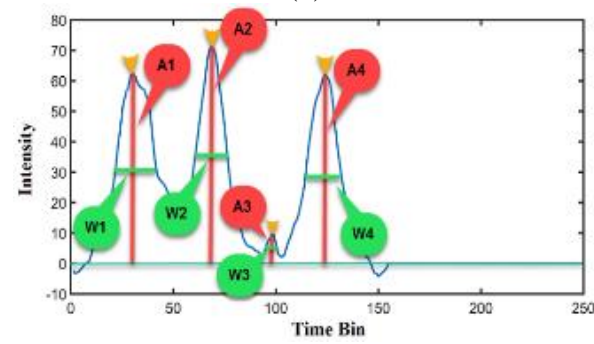

(c)

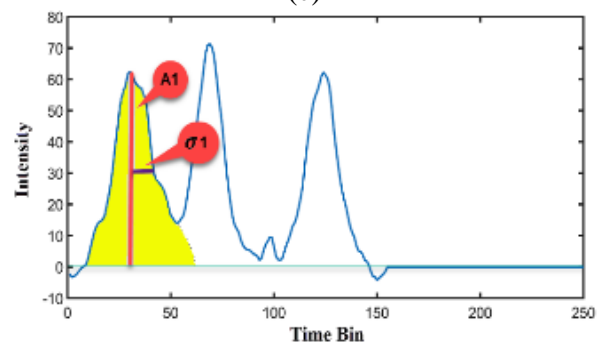

(d)

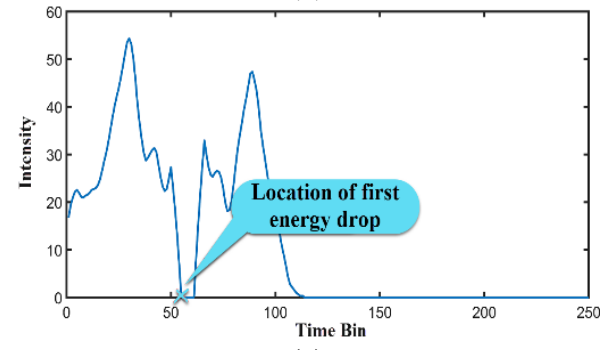

(e)

Figure 3. Generated structural features using LiDAR waveforms.

3.5 Classification using fusion of structural features of full waveform LiDAR and aerial image (waveform and RGB)

In this case, structural features of full waveform LiDAR defined in section 3.4 along with the three bands of the aerial image are used for classification.

Here, normalizing features whose values are in different ranges is necessary, especially when distance classifiers are employed (Naeini et al., 2017). In this study, in order to normalize the features in each case, the stretching based method normalization which maps values between $[0,1]$ was used (Aksoy and Haralick, 2001) and the effect of normalization on each classification strategy was assessed.

\section{EXPERIMENAL RESULTS}

In this section, the results of classification with and without feature normalization based on kappa coefficient for five proposed strategies are illustrated (Figure 4). Classification map for the case of using fusion of structural features of full waveform LiDAR and aerial image is shown in Figure 5.

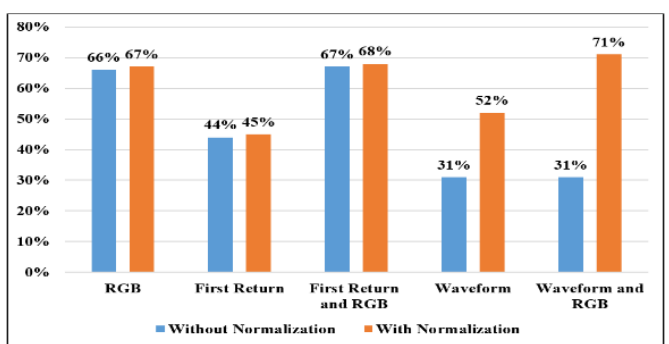

Figure 4. Classification accuracies based on kappa

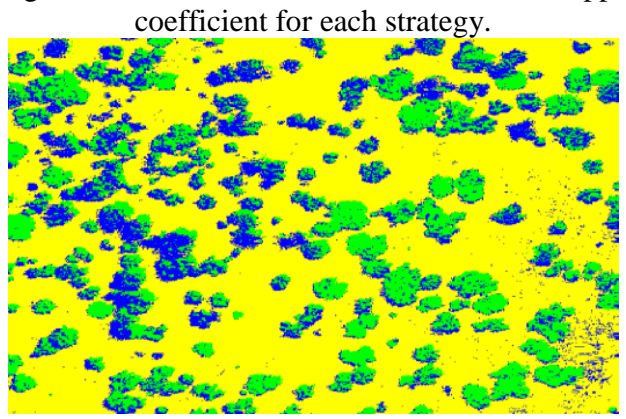

Figure 5. Classification map for the case of using fusion of structural features of full waveform LiDAR and aerial image.

As is clear from Figure 4, normalizing features leads to improvement in the classification accuracy for all five strategies. It is worth noting that in the cases which the number of features and the differences in their ranges were higher (4th and 5th cases in which structural features of LiDAR waveforms were used), normalizing features is absolutely necessary and leads to much more improvements in classification accuracy than other three cases. As it is obvious from the results, even though using nonnormalized structural features of LiDAR does not lead to high accuracy of classification result but after normalization, fusion of structural features of full waveform LiDAR and aerial image bands leads to the highest classification accuracy among five cases. After feature normalization, in the case of using just LiDAR features for classification, structural features of LiDAR waveforms improves the classification accuracy by $7 \%$, compared to using only common LiDAR features related to first return.

Finally, even though the use of only LiDAR features for classification leads to lower accuracy than using just aerial image bands, employing fusion of common LiDAR features and aerial image and fusion of structural features of full waveform LiDAR and aerial image improves the results respectively by $1 \%$ and $4 \%$ in classification accuracy when compared with using only aerial image bands.

\section{CONCLUSIONS}

LiDAR datasets, especially full waveform LiDAR data, is a valuable remote sensing source which is used effectively in numerous applications such as tree species classification. LiDAR waveform decomposition is a pre-processing step in most classification tasks which causes a part of information in waveform to be lost. In this study, we tried to use raw waveform LiDAR data for classification and assessed the potential of using structural features of non-decomposed LiDAR waveforms and its fusion with optical image for classifying a sparsely forested area. To this end, five different strategies including using RGB image, common LiDAR features, fusion of RGB image and common LiDAR features, LiDAR waveform features and fusion of RGB image and LiDAR waveform features were used for classifying the study area and the results were compared. 
The results of this study showed that normalizing features improves the classification accuracy in all five strategies but this effect is much more significant for cases in which structural feature of LiDAR waveforms is used; this is because in these cases the number of features and the differences in their ranges were higher. Using LiDAR waveform structural features leads to higher classification accuracy than using common LiDAR features coming from waveform decomposition. This is due to the fact that LiDAR structural features presents more information about vertical structure of trees than common LiDAR features. After feature normalization, fusion of LiDAR waveform structural features and aerial image led to a $3 \%$ and $4 \%$ improvement in classification accuracy when compared with using only aerial image bands and fusion of common LiDAR features and aerial image, respectively. After all, we conclude that using non-decomposed LiDAR waveforms and generating suitable structural features in classification tasks can lead to better results than using only optical images or common LiDAR features. Future attempts should be focused on extracting more effective LiDAR waveform structural features and its fusion with other sources such as hyperspectral images for classification tasks.

\section{ACKNOWLEDGEMENTS}

The National Ecological Observatory Network is a project sponsored by the National Science Foundation and managed under cooperative agreement by NEON, Inc. This material is in part based upon work supported by the National Science Foundation under Grant No. DBI-0752017. Any opinions, findings, and conclusions or recommendations expressed in this material are those of the authors and do not necessarily reflect the views of the National Science Foundation.

\section{REFERENCES}

Abed, F.M.A., 2012. Calibration of full-waveform airborne laser scanning data for 3D object segmentation.

Aksoy, S., Haralick, R.M., 2001. Feature normalization and likelihood-based similarity measures for image retrieval. Pattern recognition letters $22,563-582$.

Boiman, O., Shechtman, E., Irani, M., 2008. In defense of nearest-neighbor based image classification, 2008 IEEE Conference on Computer Vision and Pattern Recognition. IEEE, pp. 1-8.

Brandtberg, T., 2002. Individual tree-based species classification in high spatial resolution aerial images of forests using fuzzy sets. Fuzzy Sets and Systems 132, 371-387.

Cao, L., Coops, N.C., Innes, J.L., Dai, J., Ruan, H., She, G., 2016. Tree species classification in subtropical forests using smallfootprint full-waveform LiDAR data. International Journal of Applied Earth Observation and Geoinformation 49, 39-51.

Chauve, A., Mallet, C., Bretar, F., Durrieu, S., Deseilligny, M.P., Puech, W., 2008. Processing full-waveform lidar data: modelling raw signals, International archives of photogrammetry, remote sensing and spatial information sciences 2007, pp. 102-107.

Fassnacht, F.E., Latifi, H., Stereńczak, K., Modzelewska, A., Lefsky, M., Waser, L.T., Straub, C., Ghosh, A., 2016. Review of studies on tree species classification from remotely sensed data. Remote Sensing of Environment 186, 64-87.
Foody, G.M., 2003. Remote sensing of tropical forest environments: towards the monitoring of environmental resources for sustainable development. International journal of remote sensing 24, 4035-4046.

Giuliani, G., Dao, H., De Bono, A., Chatenoux, B., Allenbach, K., De Laborie, P., Rodila, D., Alexandris, N., Peduzzi, P., 2017. Live Monitoring of Earth Surface (LiMES): A framework for monitoring environmental changes from Earth Observations. Remote sensing of environment 202, 222-233.

Heinzel, J., Koch, B., 2011. Exploring full-waveform LiDAR parameters for tree species classification. International Journal of Applied Earth Observation and Geoinformation 13, 152-160.

Hollaus, M., Mücke, W., Höfle, B., Dorigo, W., Pfeifer, N., Wagner, W., Bauerhansl, C., Regner, B., 2009. Tree species classification based on full-waveform airborne laser scanning data. Proceedings of SILVILASER, 14-16.

Hovi, A., Korhonen, L., Vauhkonen, J., Korpela, I., 2016. LiDAR waveform features for tree species classification and their sensitivity to tree-and acquisition related parameters. Remote sensing of environment 173, 224-237.

Hyyppä, J., Hyyppä, H., Yu, X., Kaartinen, H., Kukko, H., Holopainen, M., 2009. Forest inventory using small-footprint airborne lidar. Topographic laser ranging and scanning: principles and processing. CRC Press, Taylor \& Francis London, 335-370.

Immitzer, M., Atzberger, C., Koukal, T., 2012. Tree species classification with random forest using very high spatial resolution 8-band WorldView-2 satellite data. Remote Sensing 4, 2661-2693.

Katila, M., Tomppo, E., 2001. Selecting estimation parameters for the Finnish multisource National Forest Inventory. Remote Sensing of Environment 76, 16-32.

Lim, T.-S., Loh, W.-Y., Shih, Y.-S., 2000. A comparison of prediction accuracy, complexity, and training time of thirty-three old and new classification algorithms. Machine learning 40, 203228.

Lindberg, E., Eysn, L., Hollaus, M., Holmgren, J., Pfeifer, N., 2014. Delineation of tree crowns and tree species classification from full-waveform airborne laser scanning data using 3-D ellipsoidal clustering. IEEE Journal of Selected Topics in Applied Earth Observations and Remote Sensing 7, 3174-3181. Mallet, C., Bretar, F., 2009. Full-waveform topographic lidar: State-of-the-art. ISPRS Journal of photogrammetry and remote sensing 64, 1-16.

Naeini, A.A., Babadi, M., Homayouni, S., 2017. ASSESSMENT OF NORMALIZATION TECHNIQUES ON THE ACCURACY OF HYPERSPECTRAL DATA CLUSTERING. International Archives of the Photogrammetry, Remote Sensing \& Spatial Information Sciences 42.

Reitberger, J., Krzystek, P., Stilla, U., 2006. Analysis of full waveform lidar data for tree species classification. International Archives of Photogrammetry, Remote Sensing and Spatial Information Sciences 36, 228-233.

Reitberger, J., Krzystek, P., Stilla, U., 2008. Analysis of full waveform LIDAR data for the classification of deciduous and 
coniferous trees. International journal of remote sensing 29 , $1407-1431$.

Reitberger, J., Schnörr, C., Krzystek, P., Stilla, U., 2009. 3D segmentation of single trees exploiting full waveform LIDAR data. ISPRS Journal of Photogrammetry and Remote Sensing 64, 561-574.

Suárez, J.C., Ontiveros, C., Smith, S., Snape, S., 2005. Use of airborne LiDAR and aerial photography in the estimation of individual tree heights in forestry. Computers \& Geosciences 31 , 253-262.

Vaughn, N.R., Moskal, L.M., Turnblom, E.C., 2011. Fourier transformation of waveform Lidar for species recognition. Remote Sensing Letters 2, 347-356.

Vaughn, N.R., Moskal, L.M., Turnblom, E.C., 2012. Tree species detection accuracies using discrete point lidar and airborne waveform lidar. Remote Sensing 4, 377-403.

Yao, W., Krzystek, P., Heurich, M., 2012. Tree species classification and estimation of stem volume and DBH based on single tree extraction by exploiting airborne full-waveform LiDAR data. Remote Sensing of Environment 123, 368-380.

Zhang, D., Chen, S., Zhou, Z.-H., 2006. Learning the kernel parameters in kernel minimum distance classifier. Pattern Recognition 39, 133-135. 